OPCT-94-3

hep-lat/9412109

\title{
Low-Temperature Expansion of the Free Energy in ASOS Model
}

\author{
H. ARISUE \\ Osaka Prefectural College of Technology \\ Saiwai-cho, Neyagawa, Osaka 572, Japan \\ Internet:arisue@ipc.osaka-pct.ac.jp
}

December 1994

\begin{abstract}
We calculate the low-temperature series of the free energy in absolutevalue solid-on-solid (ASOS) model to order $u^{23}$ using finite-lattice method. The property of the obtained series and the behavior of their Padé approximants confirms us that the roughening transition in ASOS model is of Kosterlitz-Thouless type.
\end{abstract}




\section{Introduction}

Recently the low-temperature series in $d=3$ Ising model or equivalently strong-coupling series in $d=3 Z_{2}$ lattice gauge theory have been extended to higher orders using finite-lattice method [1, 2, 3]. This method avoids the problem involved in the graphical method, in which it is rather difficult to give the algorithm for listing all the diagrams up completely that contribute to the relevant order of the series. The low-temperature series calculated by finite-lattice method in $d=3$ Ising model are for the true inverse correlation length (which is equivalent to the mass gap in lattice gauge theory) [4], free energy [5, 6], magnetization and zero-field susceptibility [6], surface tension (which is equivalent to the string tension in lattice gauge theory) [7] and the second moment of the correlation function [8]. We should mention that, using a modification of the shadow-lattice technique, low-temperature series were obtained for free energy, magnetization and susceptibility [9], which are longer than those of reference [5, 6]. The second moment was also calculated by the same technique [10], in which exact series is shorter and estimated series is longer than that of reference [8].

As for the surface tension we [7] calculated the low-temperature series to order $u_{\text {Ising }}^{17}$ with $u_{\text {Ising }}=\exp \left(-4 \beta_{\text {Ising }}\right)$ and found that the series coefficient change their sign at the order of $u_{\text {Ising }}^{13}$. The surface tension in threedimensional Ising model or the string tension in three- or higher-dimensional lattice gauge theory suffers from the roughening transition [11, 12, 13] and it is expected to exhibit Kosterlitz-Thouless [14 type singularity like

$$
f(u)=A(u) \exp \left[-c\left(u_{r}-u\right)^{-1 / 2}\right]+B(u) .
$$

It has the essential singularity at the roughening transition point $u_{r}$. As was pointed out by Hasenbusch and Pinn [15], the sign-change of the coefficients is just the signal of the K-T type singularity. In fact if we expand the function

(1) in terms of $u$ we would obtain a series with sign-change. The order of the sign-change depends on $c$ and $u_{r}$. The Padé approximants of the lowtemperature series for the surface tension appear to converge below the order of the sign-change [16]. They, however, start to scatter just at the order of the sign-change and appears to converge again for higher orders [7]. The Padé approximants of the maximal-order series for the surface tension exhibit good convergence but their values overshoot the high precision Mote Carlo results [15]. We think that our series are yet too short for the asymptotic regime to 
be reached. Unfortunately it would be difficult to extend the series further by the presently available technique of the finite-lattice method and computer resources.

Absolute-value solid-on-solid (ASOS) model is an approximation of the interface of $d=3$ Ising model. Overhangs and disconnected parts are neglected in the former. The ASOS model is also expected to exhibit K-T type phase transition. Its free energy corresponds to the surface tension of $d=3$ Ising model and is expected to behave like (1). Hasenbusch and Pinn [15] calculated the low-temperature series for the free energy of this model to order $u^{12}$ using the finite-lattice method, extending the previous series by Weeks et al [17], and they found the expected sign-change of the coefficients at the order of $u^{11}$. They analyzed the series by Padé approximants, compared with their Monte Carlo data and obtained the results similar to the case of the surface tension in $d=3$ Ising model. They concluded that the Padé approximants are inconsistent to the Monte Carlo data. Only two terms after the sign-change, however, would be too short to make a definite conclusion.

In this paper we extend the low-temperature series for the free energy of ASOS model to order $u^{23}$ using the finite-lattice method. An improved technique is introduced to extend the series, which can also be applied to the low-temperature expansion of the spin systems whose spin-variable takes more than 2 values such as the spin- $S$ Ising model with $S \geq 1$ or the $q$-state Potts model with $q \geq 3$. The obtained longer series enables us to make a definite conclusion on the roughening transition in the ASOS model and on the convergence of the Padé approximants for the free energy.

\section{Algorithm}

Here we give the improved algorithm of the low-temperature expansion for SOS model that enables us to obtain longer series than the case when the finite-lattice method was applied naively. Let us consider two-dimensional $L_{x} \times L_{y}$ rectangular lattice $\Lambda_{0}$. The free energy density in the infinite-volume limit is given by

$$
f=-\lim _{\substack{L_{x}, L_{y} \rightarrow \infty, H_{+}, H_{-} \rightarrow \infty}} \frac{1}{L_{x} L_{y}} \ln \left[Z\left(L_{x}, L_{y} ; H_{+}, H_{-}\right)\right],
$$


with Hamiltonian

$$
Z\left(L_{x}, L_{y} ; H_{+}, H_{-}\right)=\sum_{\{h\}} \exp \left(-\beta \sum_{<i, j>}\left|h_{i}-h_{j}\right|\right)
$$

where the variable $h_{i}$ at each site $i$ is restricted to $-H_{-} \leq h_{i} \leq H_{+}$ $\left(H_{+}, H_{-}>0\right)$. The low-temperature series is calculated with respect to the expansion parameter $u=\exp (-2 \beta)$. We take the boundary condition that all the variables outside $\Lambda_{0}$ are fixed to be zero.

Let us consider the set $\{\Lambda\}$ of all two-dimensional rectangular sub-lattices of $\Lambda_{0}$. The sub-lattice $\Lambda$ is denoted by its size $l_{x} \times l_{y}$ and its position in $\Lambda_{0}$. We define $H$ of $\Lambda$ as

$$
H\left(\Lambda ; h_{+}, h_{-}\right)=-\ln \left[Z\left(\Lambda ; h_{+}, h_{-}\right)\right] .
$$

In the calculation of the partition function $Z\left(\Lambda ; h_{+}, h_{-}\right)$the variable $h_{i}$ inside the $\Lambda$ is restricted to the range of $-h_{-} \leq h_{i} \leq h_{+}\left(h_{+}, h_{-} \geq 0\right)$ and all the variables outside are fixed to be zero. We define $W$ of $\Lambda$ recursively as

$$
W\left(\Lambda ; h_{+}, h_{-}\right)=H\left(\Lambda ; h_{+}, h_{-}\right)-\sum_{\substack{\Lambda^{\prime} \subseteq \Lambda, h_{+}^{\prime} \leq h_{+}, h_{-}^{\prime} \leq h_{-}}}^{\left({ }^{\prime}\right)} W\left(\Lambda^{\prime} ; h_{+}^{\prime}, h_{-}^{\prime}\right) .
$$

Here the summation $\sum^{\left({ }^{\prime}\right)}$ implies that the $W\left(\Lambda ; h_{+}, h_{-}\right)$should be excluded in the summation. We note that $H\left(\Lambda ; h_{+}, h_{-}\right)$and $W\left(\Lambda ; h_{+}, h_{-}\right)$is independent of the position of $\Lambda$. We know

$$
\begin{aligned}
& H\left(\Lambda_{0} ; H_{+}, H_{-}\right)=\sum_{\substack{\Lambda \subseteq \Lambda 0, h_{+} \leq H_{+}, h_{-} \leq H_{-}}} W\left(\Lambda ; h_{+}, h_{-}\right) \\
& =\sum_{\substack{l_{x} \leq L_{x}, l_{y} \leq L_{y}, h_{+} \leq H_{+}, h_{-} \leq H_{-}}}\left(L_{x}-l_{x}+1\right)\left(L_{y}-l_{y}+1\right) \\
& \times W\left(l_{x} \times l_{y} ; h_{+}, h_{-}\right) .
\end{aligned}
$$

Taking the infinite-volume limit we obtain

$$
\begin{aligned}
f & =\lim _{L_{x}, L_{y} \rightarrow \infty} \frac{1}{L_{x} L_{y}} H\left(\Lambda_{0} ; H_{+}, H_{-}\right) \\
& =\sum_{l_{x}, l_{y}, h_{+}, h_{-}} W\left(l_{x} \times l_{y} ; h_{+}, h_{-}\right) .
\end{aligned}
$$


In the standard cluster expansion of the free energy for this model, a cluster is composed of polymers and each of the polymers consists of sites that are connected by nearest-neighbor bonds. A value $h_{i}(\neq 0)$ is put to each site $i$ of the polymer. We can assign to each cluster two numbers $h_{p}$ and $h_{m}$ that are the maximum of $h_{i}(\geq 0)$ and $-h_{i}(\geq 0)$, respectively, in all the sites of the polymers the cluster consists of. Then, we can prove [2] that the Taylor expansion of $W\left(l_{x} \times l_{y} ; h_{+}, h_{-}\right)$with respect to $u$ includes the contribution from all the clusters of polymers in the standard cluster expansion that have $h_{p}=h_{+}$and $h_{m}=h_{-}$and that can be embedded into $l_{x} \times l_{y}$ lattice but cannot be embedded into any of its rectangular sub-lattices. The series expansion of $W\left(l_{x} \times l_{y} ; h_{+}, h_{-}\right)$starts from the order $u^{n}$ with $n=l_{x}+l_{y}+2\left(h_{+}+h_{-}\right)-2$. So, to obtain the expansion series to order $u^{N}$, we should take into account all the rectangular lattices that satisfy $l_{x}+l_{y}+2\left(h_{+}+h_{-}\right)-2 \leq N$ in the summation of eq. (17) and evaluate each of the $W$ 's to order $u^{N}$.

All the above algorithm can be coded on the computer and most of the CPU time and main memory are used for the evaluation of the relevant partition functions $Z\left(l_{x} \times l_{y} ; h_{+}, h_{-}\right)$to order $u^{N}$. The partition functions are calculated using the transfer matrix formalism based on building up finitesize lattices one site at a time [18]. The necessary CPU time and memory are proportional to $N l_{x} l_{y}\left(h_{+}+h_{-}+1\right)^{\min \left(l_{x}, l_{y}\right)}$ and $N\left(h_{+}+h_{-}+1\right)^{\min \left(l_{x}, l_{y}\right)}$, respectively. In table [1] we list the maximum $M_{i m p}(N)$ of this factor $N\left(h_{+}+h_{-}+1\right)^{\min \left(l_{x}, l_{y}\right)}$ for the necessary memory in calculating the series to order $u^{N}$ by this improved algorithm.

It is quite natural to introduce the partition function for the finite-size lattice with the range of the variable $h_{i}$ restricted as in equation (田). It comes from the analogy with the algorithm of the low-temperature expansion for the surface tension in $d=3$ Ising model by the finite-lattice method [7]. We should stress that it enables us to calculate much longer series than the case when we would apply the finite-lattice method naively by considering the ASOS model as a two-dimensional spin system. In the latter case we would define

$$
H(\Lambda)=-\ln [Z(\Lambda)]
$$

and

$$
W(\Lambda)=H(\Lambda)-\sum_{\Lambda^{\prime} \subset \Lambda} W\left(\Lambda^{\prime}\right)
$$

for the rectangular $l_{x} \times l_{y}$ lattice $\Lambda$. Here the variable $h_{i}$ takes all integer 
values $-\infty \leq h_{i} \leq+\infty$ in the calculation of the partition function $Z(\Lambda)$. Then the free energy density in the infinite-volume limit would be given by

$$
f=\sum_{l_{x}, l_{y}} W\left(l_{x} \times l_{y}\right) .
$$

For the expansion series to order $u^{N}$, we should take into accout all the rectangular lattices that satisfy $l_{x}+l_{y} \leq N$ in the summation of equation (10). The necessary CPU time and memory to evaluate each $Z(\Lambda)$ to order $u^{N}$ for $l_{x} \times l_{y}$ lattice is proportional to $N l_{x} l_{y}(2[N / 2]+1)^{\min \left(l_{x}, l_{y}\right)}$ and $N(2[N / 2]+1)^{\min \left(l_{x}, l_{y}\right)}$, respectively. In table 1 we also list the maximum $M_{\text {naive }}(N)$ of this factor $N(2[N / 2]+1)^{\min \left(l_{x}, l_{y}\right)}$ for the necessary memory in calculating the series to order $u^{N}$ by this naive algorithm. We can see that the necessary memory in the improved algorithm is much smaller than in the naive algorithm.

We note that the improved algorithm above can be used in the finitelattice method of the low-temperature expansion for the spin systems whose spin variable takes more than 2 values such as the spin- $S$ Ising model with $S \geq 1$ or the $q$-state Potts model with $q \geq 3$.

\section{Series}

We have calculated the expansion series for the free energy $f$ of ASOS model to order $u^{23}$. The calculation was performed on FACOM-VP2600 at Kyoto University Data Processing Center. Used main memory was about 80Mbyte and total CPU time was about 25 minutes. The obtained coefficients for the free energy are listed in table 2, where $a_{n}$ is defined as

$$
f=\sum_{n} a_{n} u^{n} .
$$

We have checked that each of the $W\left(l_{x} \times l_{y} ; h_{+}, h_{-}\right)$'s in equation (7) starts from the correct order in $u$ discussed in the previous section. The series to order $u^{12}$ for ASOS model coincides with those obtained by Hasenbusch and Pinn [15] and we have obtained new 11 terms. Also listed are the coefficients for the surface tension $\sigma$ in $d=3$ simple cubic Ising model obtained previously by the author [7]. The coefficients are defined by

$$
\sigma=2 \beta_{\text {Ising }}+\sum_{n} b_{n} u_{\text {Ising }}^{n}
$$


where $u_{\text {Ising }}=\exp \left(-4 \beta_{\text {Ising }}\right)$ is the low-temperature expansion parameter in the Ising model.

\section{Series Analysis}

It is to be noticed that the coefficients of the free energy in ASOS model are all negative to order $u^{10}$ and their sign changes at the order of $u^{11}$ [15]. We find no another change of sign in higher orders to $u^{23}$. This property of the sign-change is common to the surface tension in $d=3$ Ising model. As was pointed out in reference 15 it supports the claim that the roughening transition of these models is of Kosterlitz-Thouless type. Let us consider a $\mathrm{K}-\mathrm{T}$ type function and its expansion in $u$ as

$$
f^{K T}(u)=A u^{2} \exp \left[-c\left(u_{r}-u\right)^{-1 / 2}\right]=\sum_{n} a_{n}^{K T} u^{n}
$$

and fit this expansion to the expansion of the free energy (11) of ASOS model, so that the ratios of their successive coefficients coincide as $a_{n} / a_{n-1}=$ $a_{n}^{K T} / a_{n-1}^{K T}$ for $n=11$ and $n=23$, respectively. The order $n=11$ is just where the change of sign occurs and $n=23$ is the highest order of our series. Then we obtain $u_{r}=0.214$ and $c=0.533$. In table 3 we list the ratios $a_{n} / a_{n-1}$ for the free energy and the fitted K-T type function. We note that the ratios agree with each other within 3 per cent for the range of $n=6$ to 23 . Furthermore the fitted value of $u_{r}$ is not far from the predicted roughening transition point for ASOS model as $u_{r}=0.207(9)$ from the series analysis of the surface width [19 and $u_{r}=0.1994(1)$ from the Monte Carlo renormalization-group analysis [20]. The difference between the ratios for $n \leq 5$ would come from the regular background term in the free energy, whose contribution can be expected to become negligible rapidly for higher orders compared with the contribution from the singular term.

Next we will try the Padé approximation for the free energy $f(u)$ and the internal energy $E(u)=d f / d \beta=-2 u f^{\prime}(u)$, although the free energy is expected to behave like equation (11) and one might think that the Padé approximation would not be appropriate for such a function. The authors of reference [15] stressed the discrepancy between the Padé approximants of the internal energy to order $u^{12}$ and their Monte Carlo data. Their series are, however, too short to give a definite conclusion. 
In figures 1 and 2 we show the truncated series of the free energy and its Padé approximants for each order $n$ of the series in $\beta=0.85(u=0.1827)$ and $\beta=0.81(u=0.1979)$, respectively. The latter point of $\beta$ is very close to the predicted roughening transition point 20]. As for the Padé approximants we plot the $[L, M]$ approximants $(L+M=n-2)$ with $(n-2) / 4 \leq L \leq 3(n-2) / 4$. Defective approximants that have poles on the real axis of $u$ in the range of $0 \leq u \leq 0.205$ are excluded. The Padé approximants appear to converge once to some value for the shorter series. They begin to scatter just around the order of the sign-change and they converge again for higher order series but to another value. The convergence is so good in higher orders. The convergence of the truncated series is, on the other hand, much slower than the Padé approximants. In figure 3 we also give the behaviour of the Padé approximants together with the truncated series of the free energy in $\beta$ for the highest order series. From these figures we can conclude that the Padé approximation works well for the long enough series of the free energy of ASOS model even very close to the predicted roughening transition point. This situation of the Padé approximants for the free energy of ASOS model is quite similar to the case for the surface tension in $d=3$ Ising model [16, , ], in which presently available series has only 5 terms after the sign-change. We can expect that the higher order series for the surface tension in $d=3$ Ising model will also give Padé approximants converging to the Monte Carlo data and the present discrepancy [15] will be resolved.

In figures 4 and 5 we show the truncated series of the internal energy and its Padé approximants for each order $n$ of the series for $\beta=0.85$ and $\beta=0.81$, respectively. We see that the behaviors of the truncated series and its Padé approximants in the order $n$ are similar to those for the free energy given in figures 1 and 2 . The convergence of the Padé approximants is good for higher orders, although the convergence for $\beta=0.81$ is slower than in the case of $\beta=0.85$. On the contrary, the convergence of the truncated series is much slower than the Padé approximants. In figure 6 and table 1 we also give the behaviour of the Padé approximants for the internal energy versus $\beta$ for the highest order series. The Padé approximants for the highest order series are consistent with the Monte Carlo data [15] even at $\beta=0.81$ very close to the roughening transition point.

One's question might be why the naive Padé approximation, which is the ratio of two polynomials, is so good even close to the roughening transition point in spite of that the free energy is expected to have essential singularity 
as in equation (1). To clarify the reason we examine the Padé approximation for a test function

$$
g(x)=\exp \left[-(1-x)^{-1 / 2}\right]+\exp (x)
$$

The first term has the essential singularity at $x=1$ and the second term is a regular background. In figures 7 and 8 we plot the exact function, the 23rd order truncated series and its Padé approximants of the test function $g(x)$ and of its derivative $x g^{\prime}(x)$, respectively. As for the test function itself, we can see that the Padé approximants exhibit so good convergence and give the values that are consistent with the exact function for $x \leq 0.98$. As for the derivative, we observe that the Padé approximants converge and follow the exact function well for $x \leq 0.95$. The derivative shows worse convergence than the test function itself, which would be because the derivative has the power singularity multiplied by the essential singularity. The truncated series, on the contrary, starts to deviate from the exact function much earlier than the Padé approximants. We also plot in figures 9 and 10 the exact value, truncated series and its Padé approximants of the free energy and the internal energy, respectively, for each order $n$ of the series in $x=0.98$. The situation of the test function in figures 7 and 9 is quite similar to that of the free energy of ASOS model in figures 3 and 2 and the situation of the derivative in figures 8 and 10 is quite similar to the internal energy of ASOS model in figures 6 and 5, respectively. We have examined several other test functions that have the same structure as equation (11) and the situations are all similar to the case of the test function $g(x)$. We can conclude that the Padé approximants of the function like equation (11), which involves an essential singularity, converges to the correct value even very close to the singularity point, if we use the expansion series in high enough order.

\section{Summary}

We have calculated the low-temperature series for the free energy of ASOS model to order $u^{23}$ using the finite-lattice method. An improved technique has been used to extend the series, which can also be applied to the lowtemperature expansion of the spin systems whose spin-variable takes more than 2 values. The property of the obtained series and the behavior of their 
Padé approximants have confirmed us that the roughening transition in the ASOS model is of Kosterlitz-Thouless type.

\section{Acknowledgements}

The author would like to thank K. Tabata, K. Pinn, M. Hasenbusch, G. Münster and P. Weisz for valuable discussions. 


\section{References}

[1] T. de Neef and I. G. Enting, J. Phys. A10 (1977) 801;

I. G. Enting, J. Phys. A11 (1978) 563; Aust. J. Phys. 31 (1978) 515

[2] H. Arisue and T. Fujiwara, Prog. Theor. Phys. 72 (1984) 1176; Preprint RIFP-588 (1985 unpublished);

H. Arisue, Nucl. Phys. B (Proc. Suppl.) 34 (1994) 240

[3] M. Creutz, Phys. Rev. B43 (1991) 10659

[4] H. Arisue and T. Fujiwara, Nucl. Phys. B285[FS19] (1987) 253;

H. Arisue and K. Tabata, Phys. Letters B322 (1994) 224

[5] G. Bhanot, M. Creutz, J. Lacki, Phys. Rev. Lett. 69 (1992) 1841

[6] A. J. Guttmann and I. G. Enting, J. Phys. A26 (1993) 807

[7] H. Arisue, Phys. Letters B313 (1993) 187

[8] H. Arisue and K. Tabata, Preprint OPCT-94-1 (hep-lat/9407023) to appear in Nucl. Phys. B

[9] C. Vohwinkel, Phys. Letters B301 (1993) 208

[10] C. Vohwinkel and P. Weisz (1991 unpublished)

[11] J. D. Weeks, G. H. Gilmer and H. J. Leamy, Phys. Rev. Lett. 31 (1973) 549

[12] A. Hasenfratz, E. Hasenfratz and P. Hasenfratz, Nucl. Phys. B180[FS2] (1981) 353

[13] C. Itzykson, M. E. Peskin and J. B. Zuber, Phys. Letters B95 (1980) 259

[14] J.M. Kosterlitz and D.J. Thouless, J. Phys. C 6 (1973) 1181;

J.M. Kosterlitz, J. Phys. C 7 (1974) 1046

[15] M. Hasenbusch and K. Pinn, Physica A203 (1994) 189

[16] L. J. Shaw and M. E. Fisher, Phys. Rev. A39 (1989) 2189 
[17] J. D. Weeks, G. H. Gilmer and H. J. Leamy, (unpublished, cited in Shaw and Fisher)

[18] I. G. Enting, J. Phys. A13 (1980) 3713;

G. Bhanot, J. Stat. Phys. 60 (1990) 55

[19] J. Adler, Phys. Rev. B36 (1987) 2473

[20] M. Hasenbusch, M. Marcu and K. Pinn, Physica A208 (1994) 124 
Table 1: Comparison of the factors $M_{i m p}(N)$ for the improved algorithm and $M_{\text {naive }}(N)$ for the naive algorithm which the necessary memory is proportional to in calculating the series to order $u^{N}$.

\begin{tabular}{|r|r|r|}
\hline$N$ & $M_{\text {imp }}(N)$ & \multicolumn{1}{|c|}{$M_{\text {naive }}(N)$} \\
\hline 2 & 4 & 6 \\
3 & 6 & 9 \\
4 & 16 & 100 \\
5 & 20 & 125 \\
6 & 54 & 2058 \\
7 & 63 & 2401 \\
8 & 216 & 52488 \\
9 & 243 & 59049 \\
10 & 810 & 1610510 \\
11 & 891 & 1771561 \\
12 & 3072 & 57921708 \\
13 & 3328 & 62748517 \\
14 & 14336 & 2392031250 \\
15 & 15360 & 2562890625 \\
16 & 65536 & 111612119056 \\
17 & 69632 & 118587876497 \\
18 & 294912 & 5808378560022 \\
19 & 311296 & 6131066257801 \\
20 & 1562500 & 333597619564020 \\
21 & 1640625 & 350277500542221 \\
22 & 8593750 & 20961814674106394 \\
23 & 8984375 & 21914624432020321 \\
24 & 46875000 & 1430511474609375000 \\
25 & 48828125 & 1490116119384765625 \\
\hline
\end{tabular}


Table 2: The low-temperature expansion coefficients $a_{n}$ for the free energy of ASOS model and $b_{n}$ for the surface tension of 3-d Ising model.

\begin{tabular}{|r|l|l|}
\hline$n$ & \multicolumn{1}{|c|}{$a_{n}$} & \multicolumn{1}{|c|}{$b_{n}$} \\
\hline 2 & -2 & -2 \\
3 & -4 & -2 \\
4 & -10 & -10 \\
5 & -24 & -16 \\
6 & $-194 / 3$ & $-242 / 3$ \\
7 & -172 & -150 \\
8 & -452 & -734 \\
9 & $-3184 / 3$ & $-4334 / 3$ \\
10 & $-8862 / 5$ & $-32122 / 5$ \\
11 & +1712 & -10224 \\
12 & $+118604 / 3$ & $-106348 / 3$ \\
13 & +284232 & +53076 \\
14 & $+11450234 / 7$ & $+3491304 / 7$ \\
15 & $+42775116 / 5$ & $+74013814 / 15$ \\
16 & +42439230 & +27330236 \\
17 & +203605872 & +160071418 \\
18 & $+8589601858 / 9$ & \\
19 & +4397057716 & \\
20 & +19982814764 & \\
21 & $+1885469273984 / 21$ & \\
22 & $+4393383644538 / 11$ & \\
23 & +1760491870744 & \\
\hline
\end{tabular}


Table 3: Comparison of the ratio of $a_{n} / a_{n-1}$ for the free energy and $a_{n}^{K T} / a_{n-1}^{K T}$ for the fitted K-T type function $f^{K T}(u)$. The parameter for the K-T type function is $u_{r}=0.214$ and $c=0.533$.

\begin{tabular}{|r|r|r|}
\hline$n$ & $a_{n} / a_{n-1}$ & $a_{n}^{K T} / a_{n-1}^{K T}$ \\
\hline 3 & 2.000 & -2.692 \\
4 & 2.500 & 2.158 \\
5 & 2.400 & 2.510 \\
6 & 2.694 & 2.658 \\
7 & 2.660 & 2.683 \\
8 & 2.628 & 2.591 \\
9 & 2.348 & 2.321 \\
10 & 1.670 & 1.634 \\
11 & -0.966 & -0.966 \\
12 & 23.093 & 22.345 \\
13 & 7.189 & 7.029 \\
14 & 5.755 & 5.635 \\
15 & 5.230 & 5.126 \\
16 & 4.961 & 4.869 \\
17 & 4.798 & 4.717 \\
18 & 4.687 & 4.618 \\
19 & 4.607 & 4.549 \\
20 & 4.545 & 4.499 \\
21 & 4.493 & 4.461 \\
22 & 4.448 & 4.432 \\
23 & 4.408 & 4.408 \\
\hline
\end{tabular}


Table 4: Comparison of the Padé approximants of the low-temperature series and the Monte Carlo data in reference [15] for internal energy. The value of the truncated series is also listed.

\begin{tabular}{|r|l|l|l|l|l|}
\hline \multicolumn{1}{|c|}{$\beta$} & 0.85 & 0.84 & 0.83 & 0.82 & 0.81 \\
\hline$u$ & 0.1827 & 0.1864 & 0.1901 & 0.1940 & 0.1979 \\
\hline \hline truncated & 0.5917 & 0.6252 & 0.6590 & 0.6919 & 0.7222 \\
\hline Padé $[6 / 15]$ & 0.5886 & 0.6202 & 0.6509 & 0.6797 & 0.7060 \\
{$[7 / 14]$} & 0.5886 & 0.6201 & 0.6508 & 0.6792 & 0.7045 \\
{$[8 / 13]$} & 0.5886 & 0.6202 & 0.6509 & 0.6795 & 0.7054 \\
{$[9 / 12]$} & 0.5887 & 0.6203 & 0.6511 & 0.6803 & 0.7081 \\
{$[10 / 11]$} & 0.5887 & 0.6203 & 0.6511 & 0.6803 & 0.7079 \\
{$[11 / 10]$} & 0.5887 & 0.6203 & 0.6512 & 0.6806 & 0.7089 \\
{$[12 / 9]$} & 0.5887 & 0.6203 & 0.6513 & 0.6807 & 0.7094 \\
{$[13 / 8]$} & 0.5886 & 0.6202 & 0.6509 & 0.6797 & 0.7059 \\
{$[14 / 7]$} & 0.5886 & 0.6202 & 0.6509 & 0.6796 & 0.7059 \\
{$[15 / 6]$} & 0.5886 & 0.6201 & 0.6507 & 0.6790 & 0.7039 \\
\hline $\mathrm{MC}(L=256)$ & $0.5887(2)$ & $0.6205(2)$ & $0.6518(2)$ & $0.6811(2)$ & $0.7096(2)$ \\
$\mathrm{MC}(L=128)$ & $0.5884(4)$ & $0.6203(4)$ & $0.6516(4)$ & $0.6805(4)$ & $0.7095(3)$ \\
$\mathrm{MC}(L=64)$ & $0.5874(8)$ & $0.6498(7)$ & $0.6498(7)$ & $0.6795(6)$ & $0.7074(6)$ \\
\hline
\end{tabular}




\section{Figure captions}

Fig. 1 : The values of the truncated series $(+)$ and its Padé approximants $(\diamond)$ for the free energy versus the order $n$ of the series in $\beta=0.85(u=$ 0.1827). The horizontal line represents the average of the Padé approximants for the highest order series of $n=23$.

Fig. 2 : The values of the truncated series $(+)$ and its Padé approximants $(\diamond)$ for the free energy versus the order $n$ of the series in $\beta=0.81(u=$ 0.1979). The horizontal line represents the average of the Padé approximants for the highest order series of $n=23$.

Fig. 3 : The Padé approximants $(\diamond)$ together with the truncated series (dashed line) of the free energy versus $u$ for the highest order series of $n=23$.

Fig. 4 : The values of the truncated series $(+)$ and its Padé approximants $(\diamond)$ for the internal energy versus the order $n$ of the series in $\beta=0.85$. The horizontal line represents the result of the Monte Carlo simulation in reference [15].

Fig. 5 : The values of the truncated series $(+)$ and its Padé approximants $(\diamond)$ for the internal energy versus the order $n$ of the series in $\beta=0.81$. The horizontal line represents the result of the Monte Carlo simulation in reference [15].

Fig. 6 : The Padé approximants (solid lines) together with the truncated series (dashed line) of the internal energy versus $u$ for the highest order series of $n=23$. The result of the Monte Carlo simulation $(\diamond)$ in reference [15] is also plotted.

Fig. 7 : The exact function (solid line), the 23rd order truncated series (dashed line) and its Padé approximants $(\diamond)$ of the test function $g(x)$.

Fig. 8 : The exact function (solid line), the 23rd order truncated series (dashed line) and its Padé approximants $(\diamond)$ of the derivative $x g^{\prime}(x)$ of the 
test function.

Fig. 9 : The values of the truncated series $(+)$ and its Padé approximants $(\diamond)$ for the test function $g(x)$ versus the order $n$ of the series in $x=0.98$. The horizontal line represents the exact value.

Fig. 10: The values of the truncated series $(+)$ and its Padé approximants $(\diamond)$ for the derivative $x g^{\prime}(x)$ of the test function versus the order $n$ of the series in $x=0.98$. The horizontal line represents the exact value. 
Fig.1

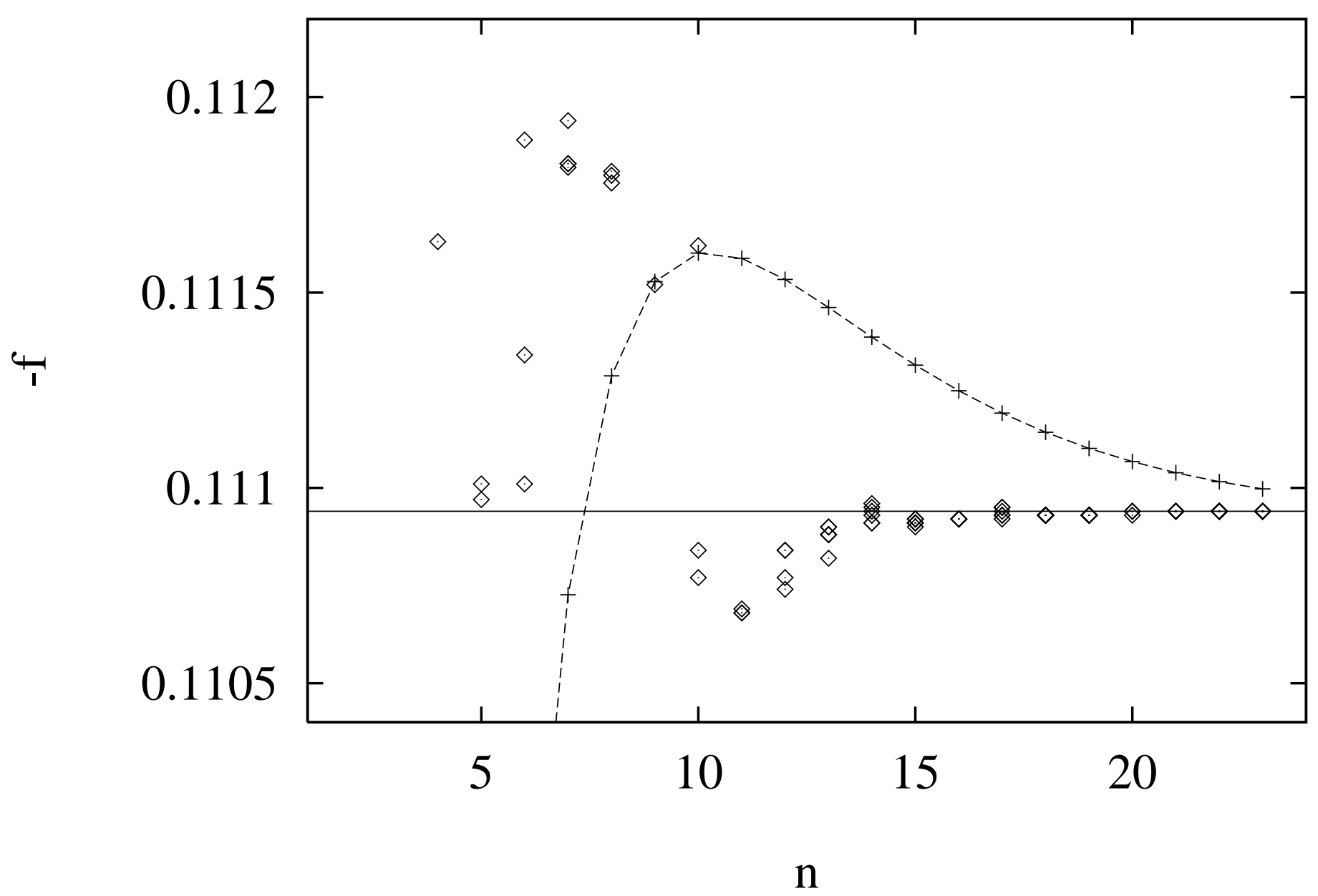


Fig. 2

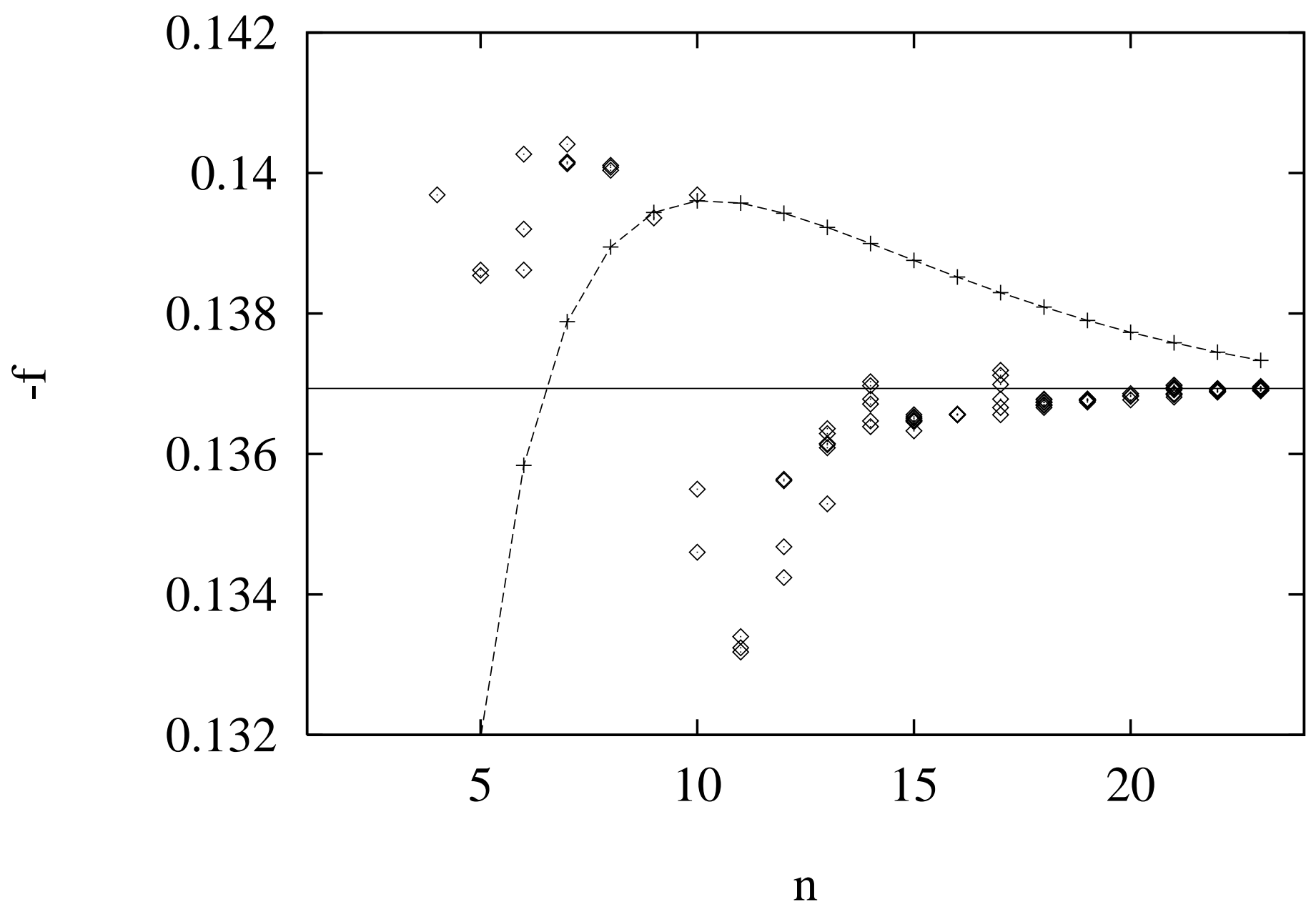


Fig.3

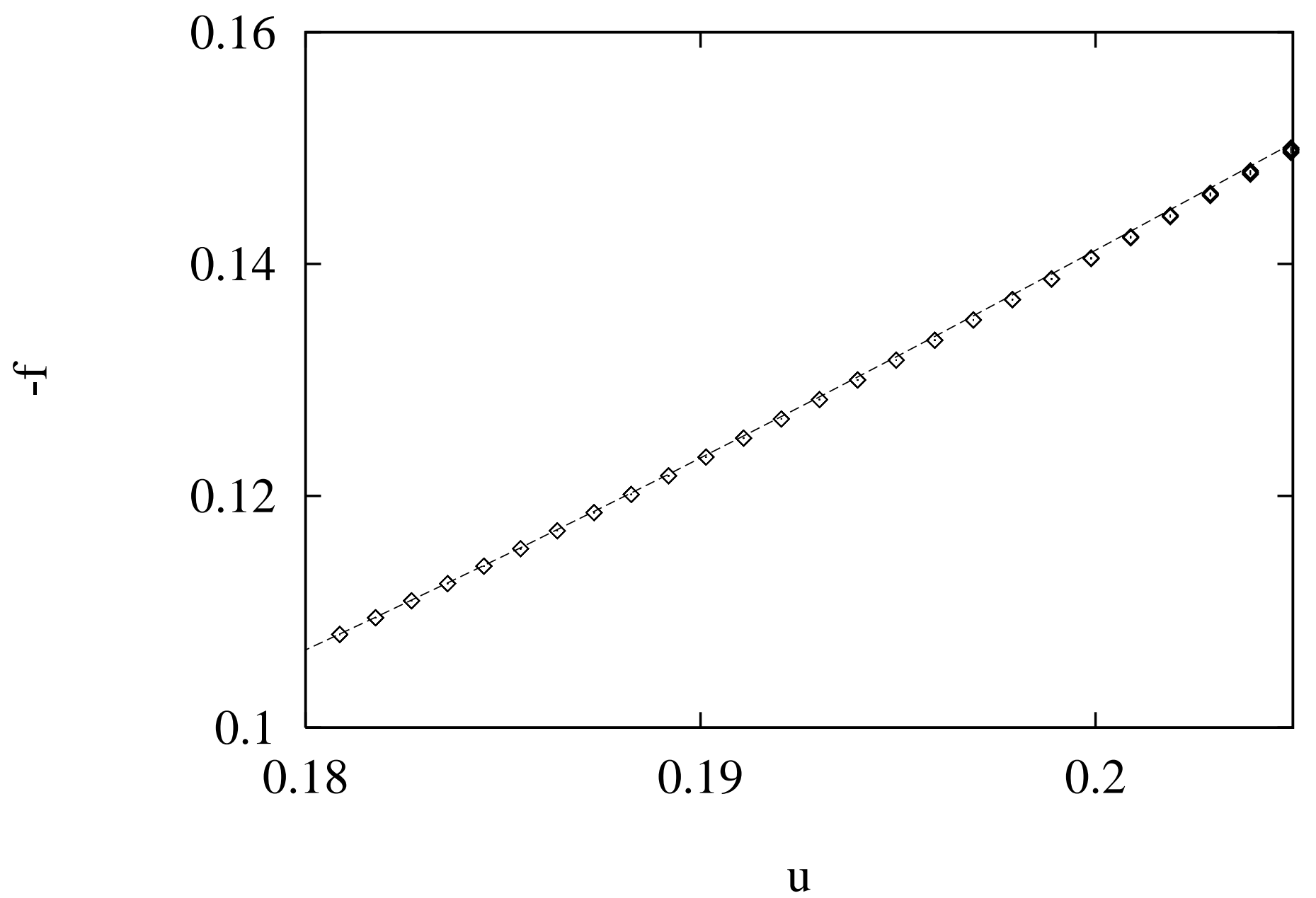


Fig.4

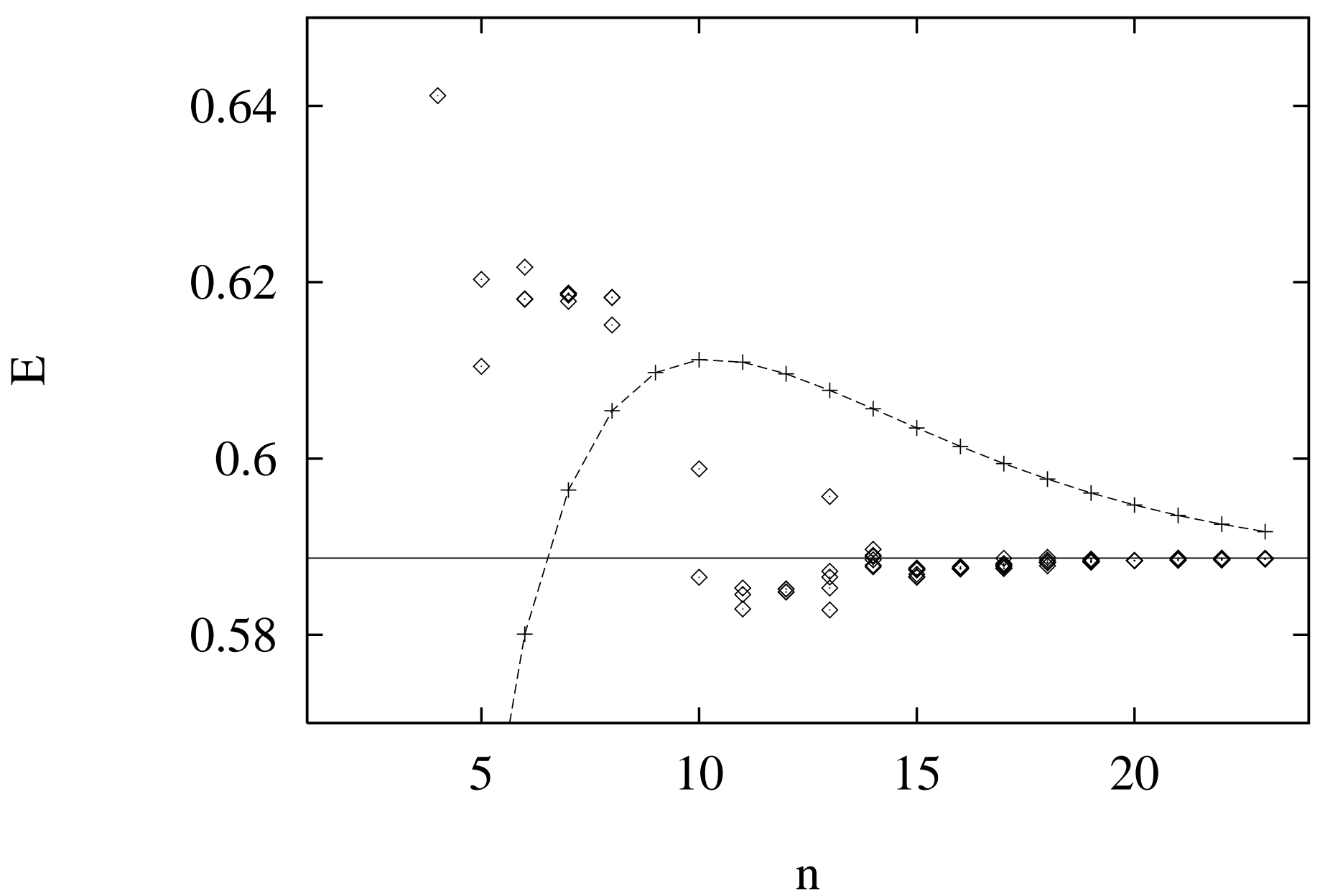


Fig.5

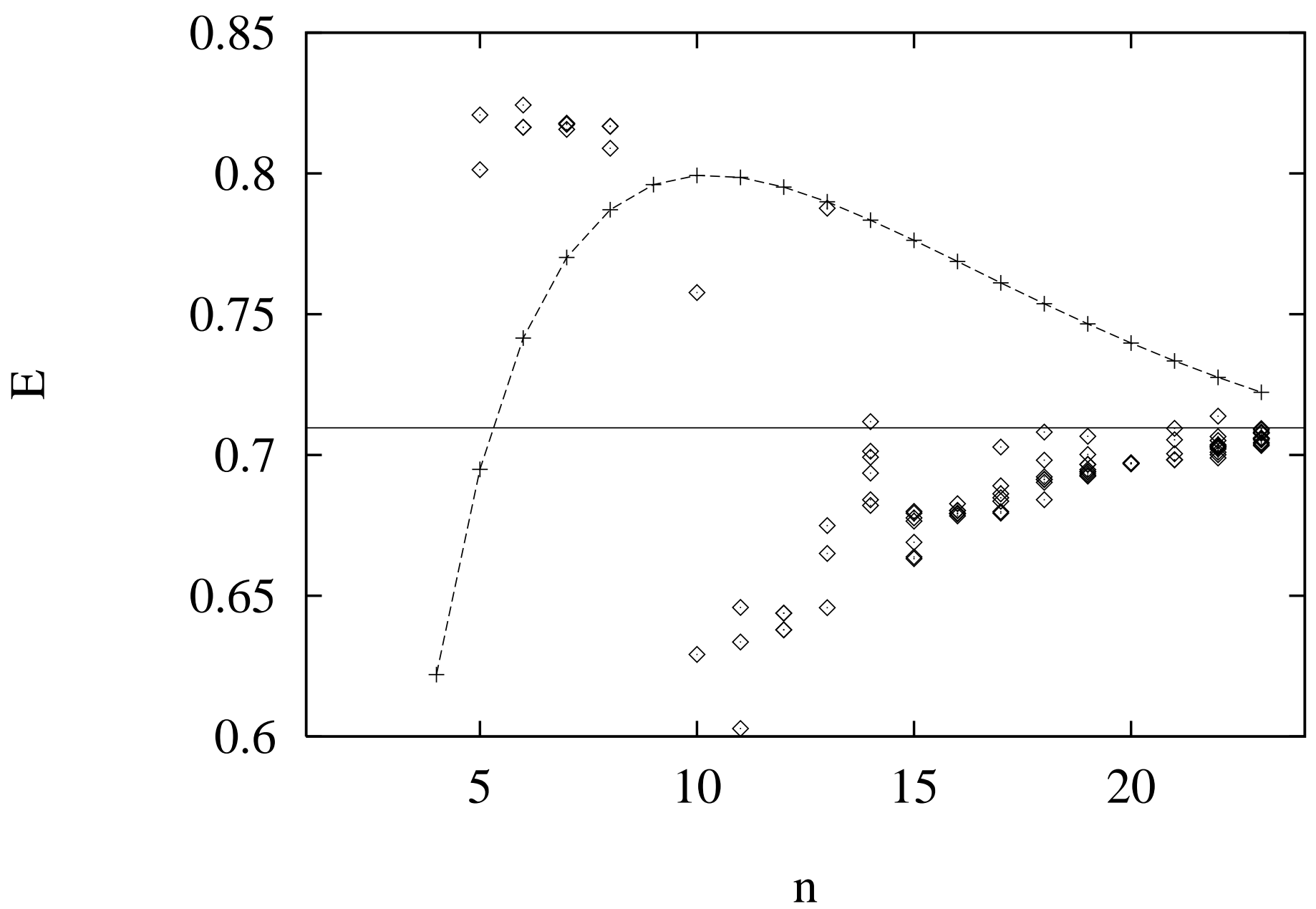


Fig.6

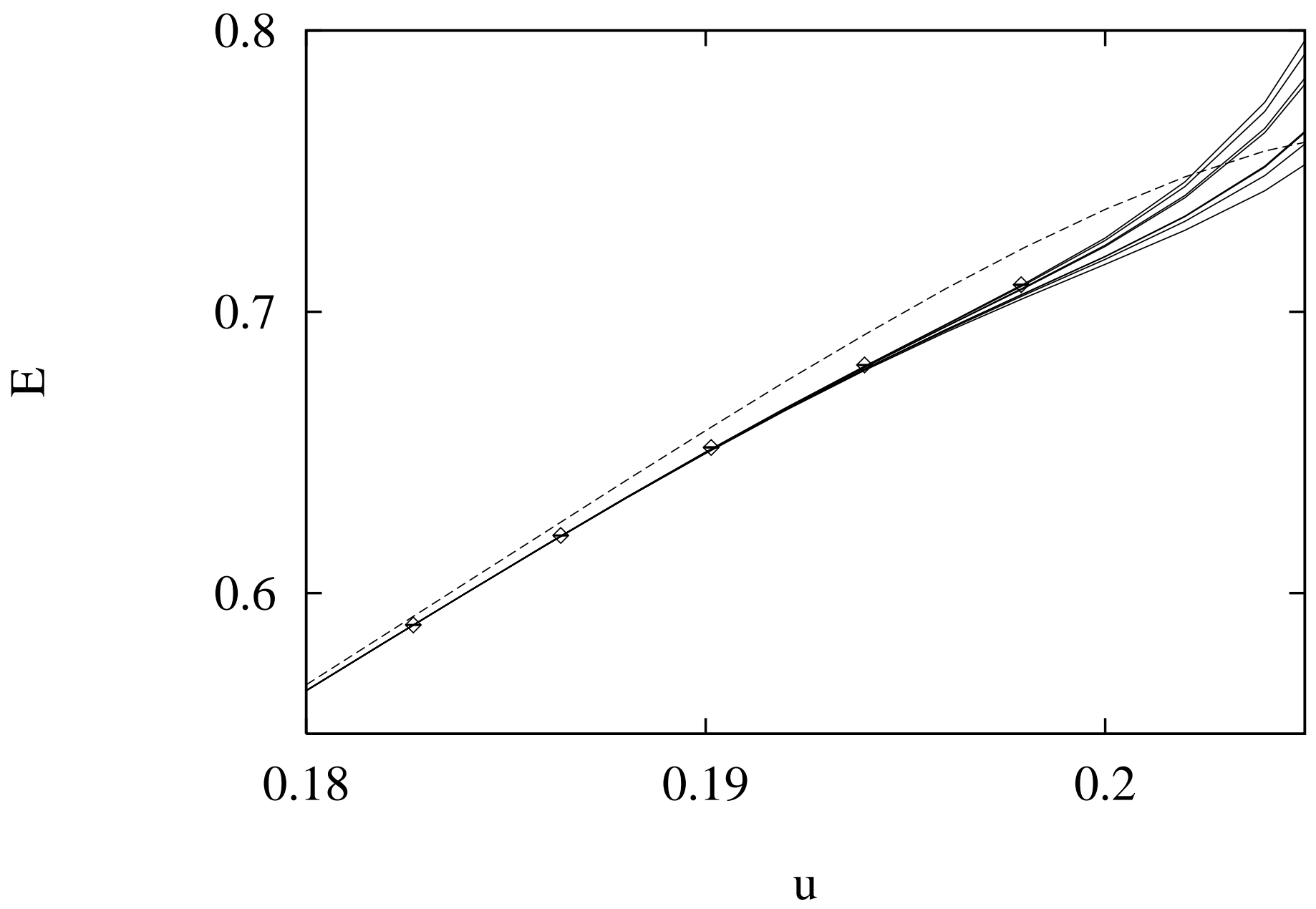


Fig.7

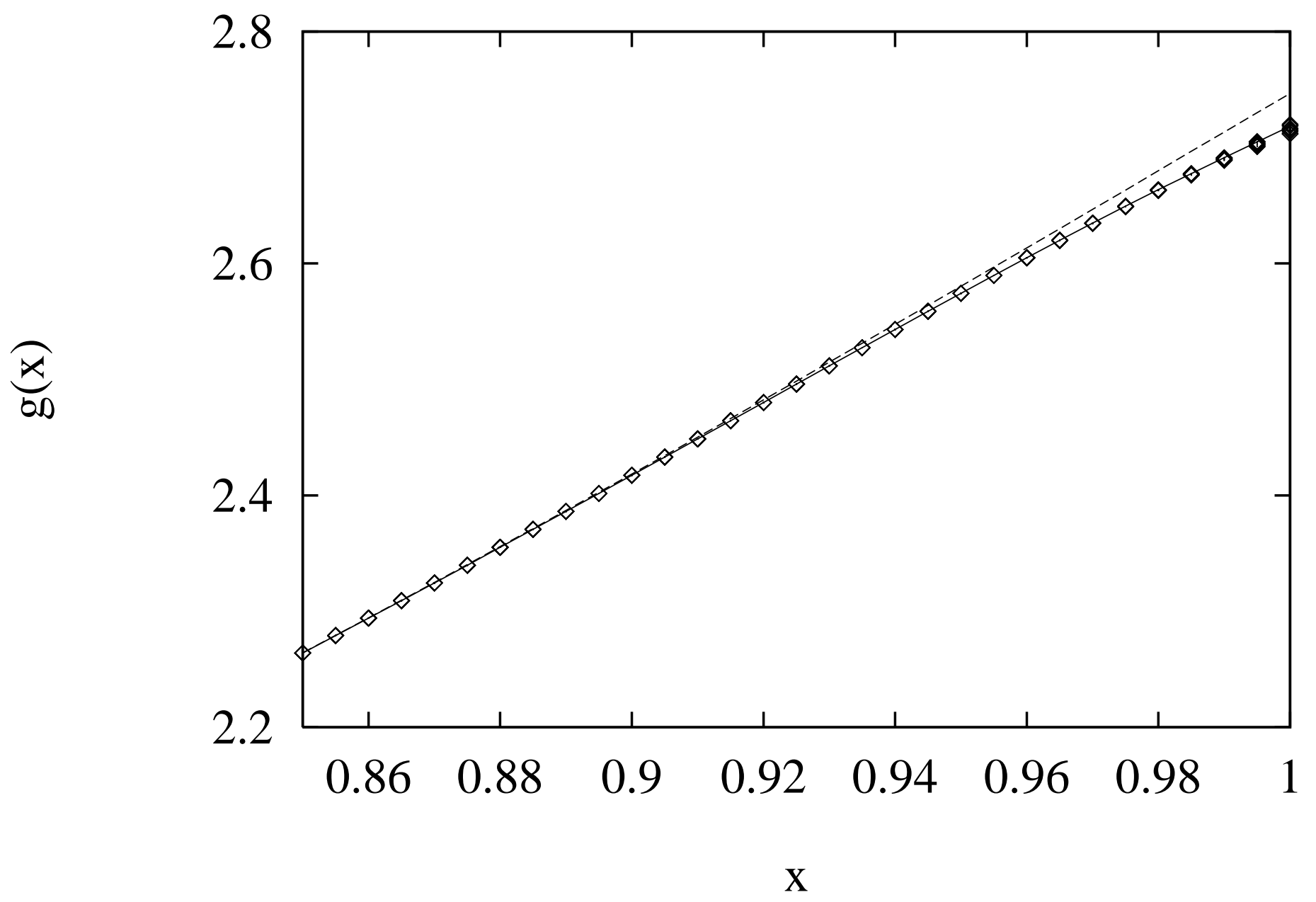


Fig.8

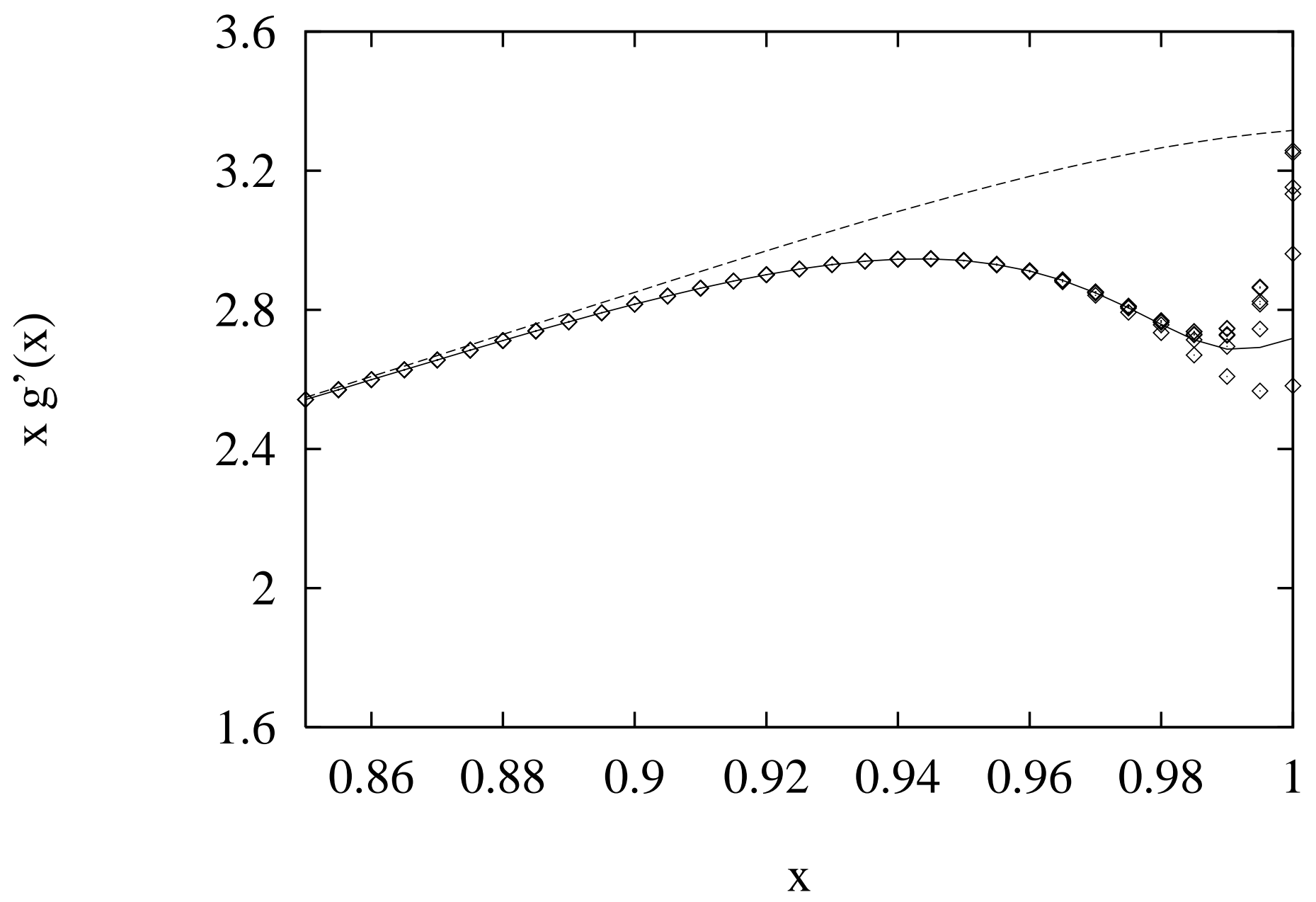


Fig.9

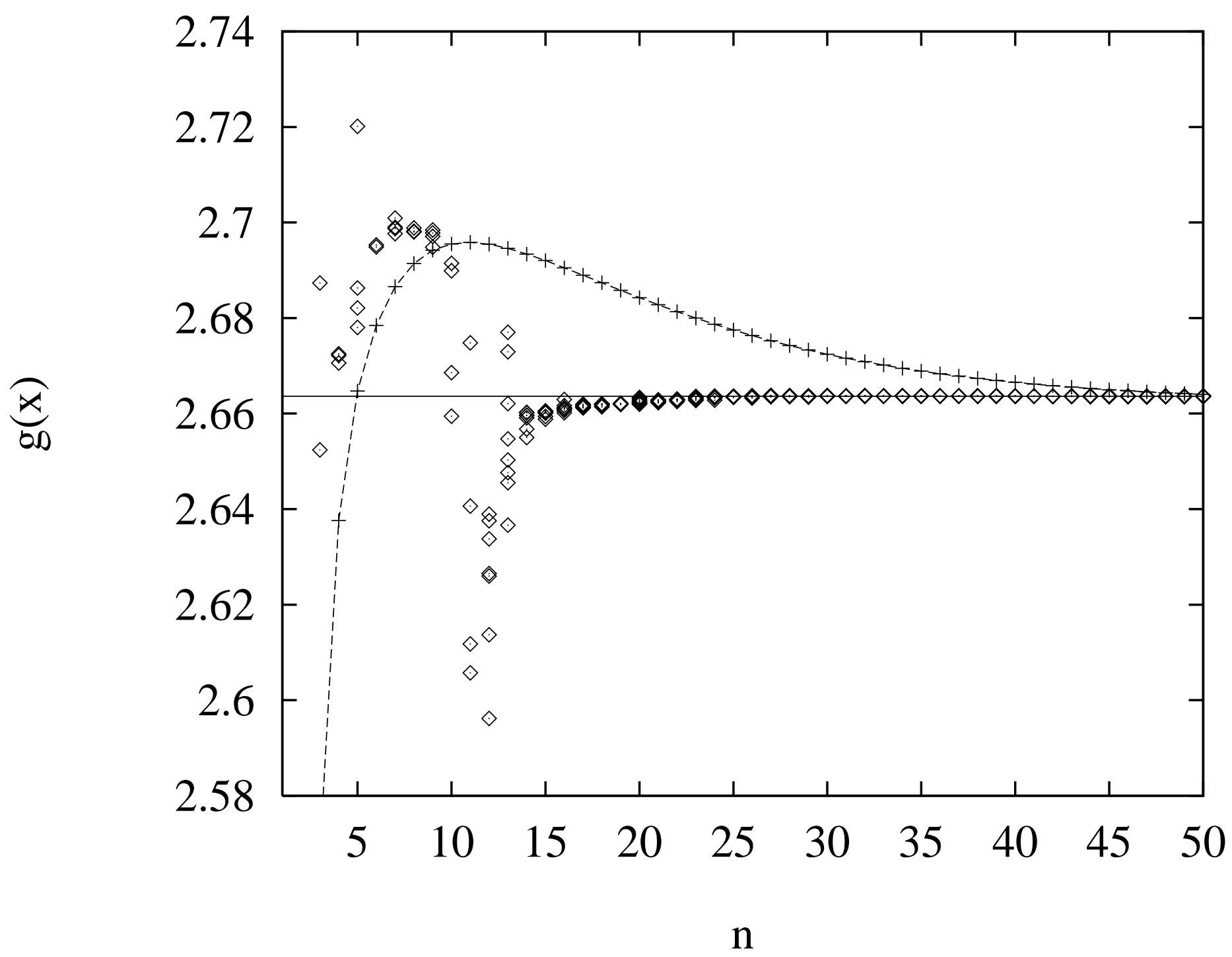


Fig.10

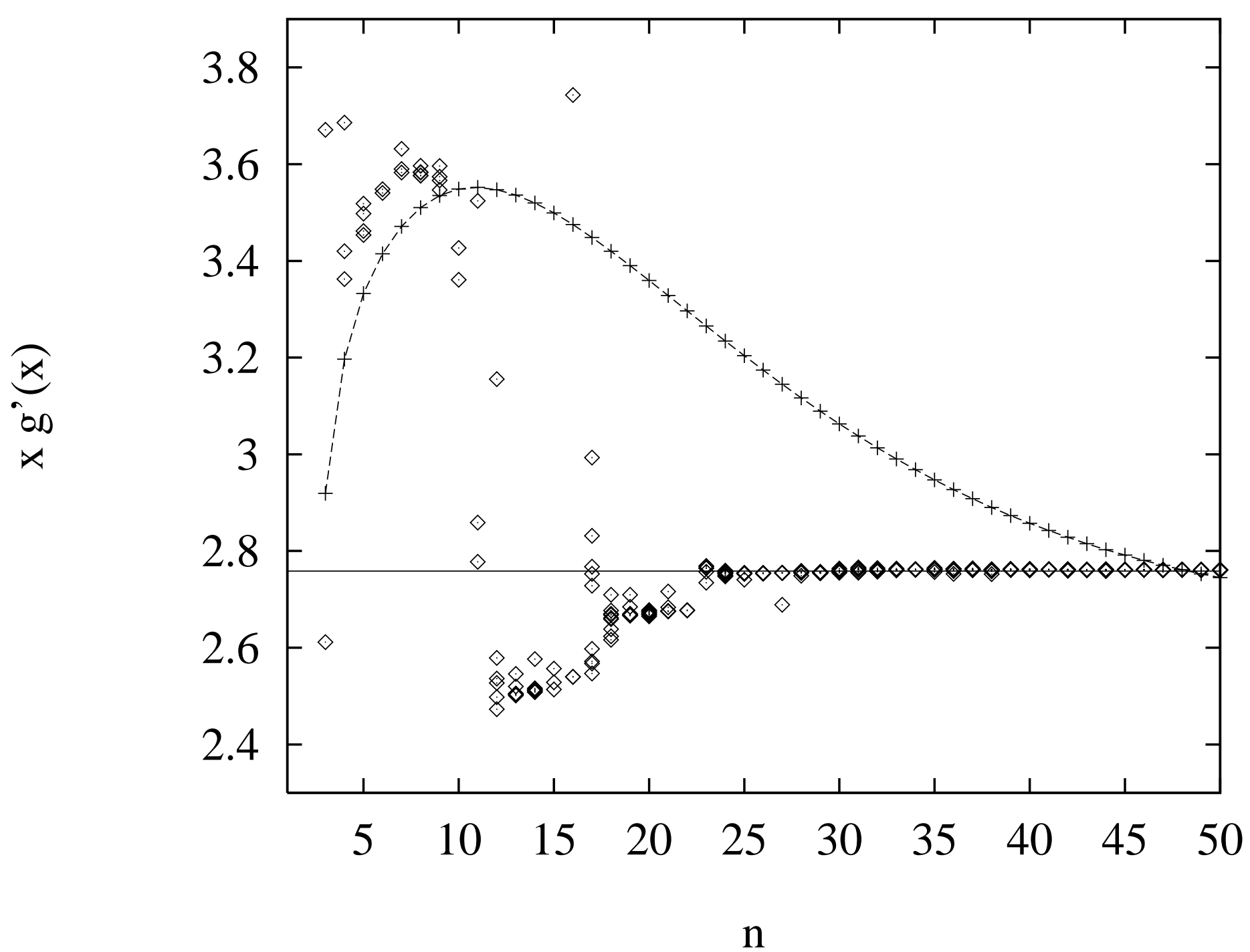


This figure "fig1-1.png" is available in "png" format from: http://arxiv.org/ps/hep-lat/9412109v1 
This figure "fig1-2.png" is available in "png" format from: http://arxiv.org/ps/hep-lat/9412109v1 
This figure "fig1-3.png" is available in "png" format from: http://arxiv.org/ps/hep-lat/9412109v1 
This figure "fig1-4.png" is available in "png" format from: http://arxiv.org/ps/hep-lat/9412109v1 
This figure "fig1-5.png" is available in "png" format from: http://arxiv.org/ps/hep-lat/9412109v1 
This figure "fig1-6.png" is available in "png" format from: http://arxiv.org/ps/hep-lat/9412109v1 
This figure "fig1-7.png" is available in "png" format from: http://arxiv.org/ps/hep-lat/9412109v1 
This figure "fig1-8.png" is available in "png" format from: http://arxiv.org/ps/hep-lat/9412109v1 
This figure "fig1-9.png" is available in "png" format from: http://arxiv.org/ps/hep-lat/9412109v1 
This figure "fig1-10.png" is available in "png" format from: http://arxiv.org/ps/hep-lat/9412109v1 